\title{
A FDTD MODEL FOR THE POST-RECEPTION SYNTHETIC FOCUSING SURFACE PENETRATING RADAR WITH MINE DETECTING APPLICATIONS.
}

\author{
R.Nilavalan, G.S.Hilton and R.Benjamin \\ Centre for Communications Research, University of Bristol, UK
}

\section{INTRODUCTION}

Surface Penetrating Radars (SPR) are extensively used in Military, Civil, Geophysical and Archaeological applications. In recent times there has been an increasing emphasis on the use of SPRs in the identification of the buried unexploded ordnance, such as anti tank mines, antipersonnel mines etc., which can be metallic or plastic in nature. Mine detection methods can be classified as destructive or non- destructive. Non destructive mine detection methods employ metal detectors, magnetometers, infrared sensors and SPRs. However, neither the metal detector nor the magnetometer can differentiate a mine from metallic debris and the infrared sensor merely notes a difference in thermal conductivity. In most battle fields the soil is contaminated by large quantity of shrapnel, metal scraps and cartridge cases which will give a high false alarm rate in the identification process. In this scenario the SPR is a promising technique for identification of both metallic and plastic mines.

Here the use of Finite Difference Time Domain (FDTD) model to analyse the SPR, which makes use of the Post Reception Synthetic Focusing (PRSF) techniques [Benjamin (1)], is presented.

\section{SURFACE PENETRATING RADARS}

\section{Present Methodology}

Surface penetrating radars are functionally similar to conventional radars. The major difference is the physical environment in which they operate. According to electromagnetic principles, when an electromagnetic wave encounters a different dielectric medium to its propagating medium, a part of the energy is reflected and the rest of the energy is transmitted into the second medium. The SPR make use of this principle to identify the objects buried in the soil; the reflected electromagnetic waves are used to identify the presence of the object. In a SPR, a modulated pulse or a single impulse is transmitted into the soil as shown in fig 1 . The reflected signals from the objects buried in the soil are received using a receiver antenna.

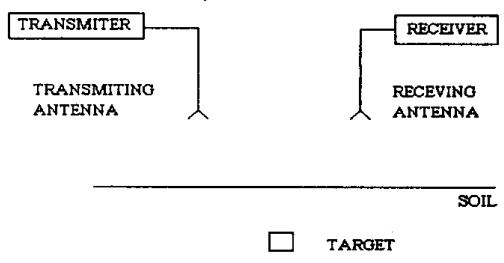

Fig 1: Surface Penetrating Radar (SPR)

The antennas are placed near or in-contact with the surface of the earth to probe the subsurface. The depth of the target is calculated depending on the time delay associated with the received pulse. The SPRs, when operating in non-contact mode, has to deal with direct coupling between elements, reflections caused at the airground interface and the reflections from various dielectric discontinuities encountered in the soil. The reflected signal from a specific target will be contaminated by reflected signals from various dielectric discontinuities which have the same path delays and this unwanted clutter makes the target identification a difficult task. Clutter encountered by SPRs is quite significant and provides a great challenge for target identification. Although SPRs have many promising features, conventional SPRs have limitations due to attenuation in ground, clutter and limited resolution.

\section{The Post-Reception Synthetic-Focusing}

The technique of Post Reception Synthetic Focusing (PRSF) [(1), Benjamin (2) and Benjamin et al (3)] can be used to overcome the limitations suffered by the conventional SPRs. In the PRSF technique, one antenna element of an antenna array is stimulated at a time and all the other relevant antenna elements are employed to record the reflected signal. These reflected signals are then used to focus on to the resolution cells in the subsurface. The PRSF technique is expected to give a fine resolution (depending on operating frequency and pulse length), high processing gain and a better performance in the sort of high clutter environment in which metal or plastic land mines are typically buried. Hence the PRSF technique is being investigated for the detection of such objects. A non-contact operation is required, since in-contact operation has the risk of setting off trip-wires and other trigger devices in mine like targets.

National Conference on Antennas and Propagation: 30 March - 1 April 1999, 
An investigation of the PRSF technique requires accurate knowledge of the reflected signals from a buried target. The theoretical field calculations associated with the Post Reception Synthetic Focusing Surface Penetrating Radar (PRSFSPR) is very complex due to:

- The antenna response

- The presence of the soil in the near field of the antenna.

- Mutual coupling between antenna elements.

- Multiple reflections between the buried target and soil air interface.

- The electrical irregularities in the soil etc.

\section{FINITE-DIFFERENCE TIME-DOMAIN METHODS}

The Finite Difference Time Domain (FDTD) method is a numerical electromagnetic modelling method, which is used to model complex electromagnetic problems. This method makes use of the Maxwell's curl equations in a finite difference form. The Maxwell's curl equations are discretised in the spatial and temporal domain and the electric and magnetic fields are calculated throughout a discreatised volume at increasing time increments. The computational domain can be obtained by dividing the problem space into unit cells, which contain three electric field components and three magnetic field components in a Cartesian co-ordinate system. Different materials are incorporated in the model by making use of their electric properties. Since the FDTD problems are mostly scattering problems and the computers have limited resources, absorbing boundaries are introduced at the problem boundary to limit the problem space. Absorbing boundaries are special boundary conditions, which are used to model the unbounded surroundings as accurately as possible [Mur (4)].

The use of FDTD techniques in complex problems have been well demonstrated. FDTD modelling is used to model Microstrip planar circuits [Shen et al (5)], Microstrip patch antennas [Hilton (6)], dipole elements and Scattering from radar targets [Taflove and Umashankar (7)].

The complex theoretical calculations of post-reception synthetic-focusing surface penetrating radar can be realistically modelled using FDTD analysis. A full FDTD model of the complete PRSFSPR system would be prohibitively complex and slow in operation. On the other hand, since all the components of a PRSFSPR system can be incorporated in the FDTD analysis, the FDTD techniques, albeit for a drastically scaled down PRSFSPR system, are particularly appropriate for a theoretical validation and evaluation of the PRSFSPR concept.
In this paper the FDTD model of a post-reception synthetic focusing surface penetrating radar is presented. The model comprises an antenna array, specimen soil and a test object that is buried within the soil. Antenna elements are placed about two wavelengths from the soil. The antenna array consists of eight printed dipole elements mounted over a ground plane that is spaced one-quarter wavelength away from the dipoles. The wide band antenna characteristics are analysed and the back-scattered fields generated by the buried test object are presented. These calculated field values are used to demonstrate the concept of post reception synthetic focusing and to establish its properties.

\section{Antenna Modelling}

A simple printed dipole antenna was modelled using FDTD analysis. The antenna element and the feed are printed on a dielectric substrate as shown in Fig 2.

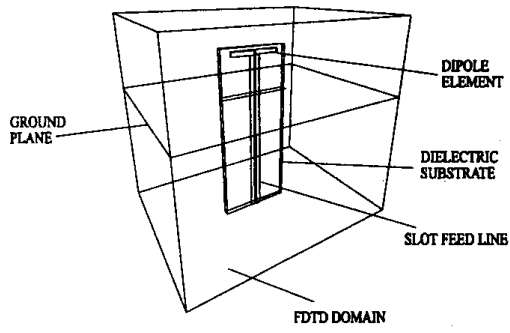

Fig 2: FDTD Model of a dipole element

The antenna element was fed by a balanced line, which passed through a ground plane (reflector). The ground plane had a rectangular hole to accommodate the feed line and the substrate. The problem space was divided into $58 \times 49 \times 55$ unit cells. The unit cells were chosen such that they properly align with the metal edges of the structure. The feed line was $114 \mathrm{~mm}$ long and was placed in such a way as to discriminate between the incident and the reflected pulse. A wideband Gaussian pulse with $250 \mathrm{ps}$ width and an unity amplitude was used to excite the element in order to observe its wide band characteristics. A probe was placed near the excitation to observe the fields on the feedline. The time step employed was $\Delta t=1.64 \mathrm{ps}$ and the simulations were performed for 15000 time steps to obtain the steady state. The computation time for this problem on a HP 900 workstation was approximately 8 hours.

The return loss of the antenna can be calculated using the fast Fourier transform of the incident and the reflected pulses. The return loss is shown in fig 3 . From this figure it can be seen that the antenna operates close to $2 \mathrm{GHz}$ and has a $-10 \mathrm{~dB}$ bandwidth of approximately $800 \mathrm{MHz}(1.8-2.6 \mathrm{GHz})$. A wide bandwidth is necessary for a SPR. 


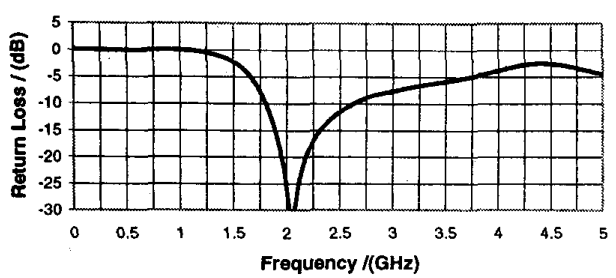

Fig 3: Return loss of the dipole antenna model

Although the antenna is to be operated close to the ground, the far field patterns can be used to determine whether the antenna is suitable for operation as a SPR antenna. The far field radiation pattern of the dipole antenna was found by post processing the near field frequency domain data at specific frequencies. The far field patterns were obtained for $1.8 \mathrm{GHz}$ and $2.6 \mathrm{GHz}$, which corresponds to the limits of the operating bandwidth of the antenna.
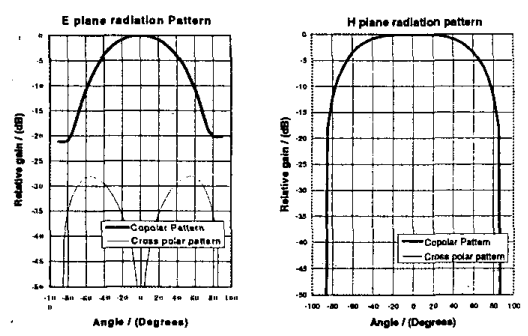

Fig 4.1: Radiation Pattern at $1.8 \mathrm{GHz}$
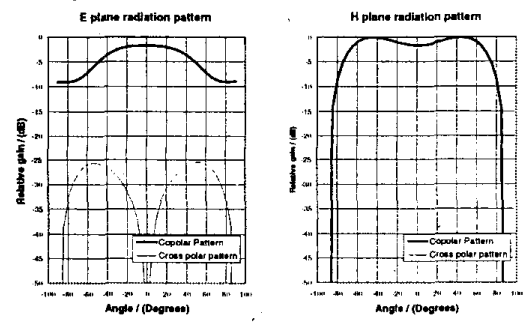

Fig 4.2: Radiation Pattern at $2.6 \mathrm{GHz}$

The corresponding far field patterns are shown in Fig 4.1 and Fig 4.2, which show that the dipole element model has a half power beamwidth of approximately \pm $45^{\circ}$ and do not significantly vary in shape over the bandwidth shown.

\section{A FDTD MODEL FOR THE PRSFSPR}

A post reception synthetic focusing SPR FDTD model is shown in Fig 5. In this model the soil was modelled with a dielectric constant of 6 (equivalent to sandy loam) and a buried object was simulated with a flat metal plate with dimensions of $0.4 \lambda \times 0.4 \lambda$ (at $1.93 \mathrm{GHz}$ ). The problem space contained $199 \times 139 \times 63$ unit cells in $x, y$, $\mathrm{z}$ directions respectively. A non-uniform mesh was used with the largest unit cell dimension being $7.5 \mathrm{~mm}$ and the smallest $0.66 \mathrm{~mm}$. Mur's $1 \mathrm{st}$ order boundary conditions [4] were used to limit the problem space. The feed was kept $263 \mathrm{~mm}$ long to avoid reflections from the antenna reaching the voltage source before it was switched off.

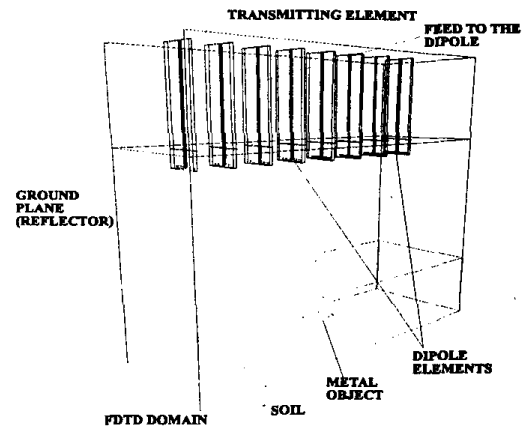

Fig 5: FDTD Model of a PRSFSPR system

The antenna array was modelled with 8 printed dipole elements, each one having the radiation characteristics described in the previous section. The first antenna element was excited with a modulated square pulse of 2ns width and $1.93 \mathrm{GHz}$ carrier and all 8 elements were used to receive the signals. The antenna array is displaced 2 free space wavelengths from the air-soil interface. The signals received at the antenna elements contain the mutual coupling between the transmitter and receiver, reflections from the air-soil interface, the reflected signals from the buried object and signals due to multiple reflections. The reflected signals from the buried target (with extra reflections) were calculated by running the same model without the buried metal plate and subtracting the received signals from the signals which were obtained with the object. The calculated reflected signals are shown in Fig 6 (for elements 1-4).
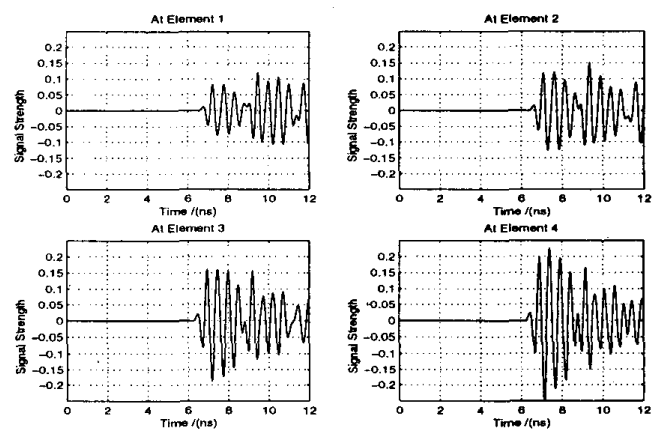

Fig 6: Reflected signals from a metal object in soil

\section{Post Reception Synthetic Focussing with the FDTD} Results

Post reception synthetic focusing is achieved by calculating the path delays associated with the different transmitting and receiving elements via the focussing point and adding the signals in phase coherence. Fig 7 and Fig 8 shows the results obtained from lateral and vertical focussing through the target co-ordinates (at a depth of $100 \mathrm{~mm}$ and lateral position of $400 \mathrm{~mm}$ ) 


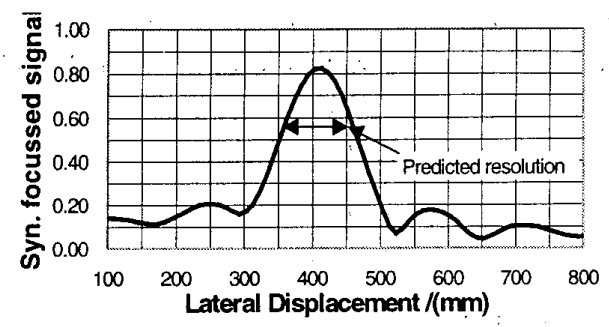

Fig 7: Lateral focussing with target at $400 \mathrm{~mm}$

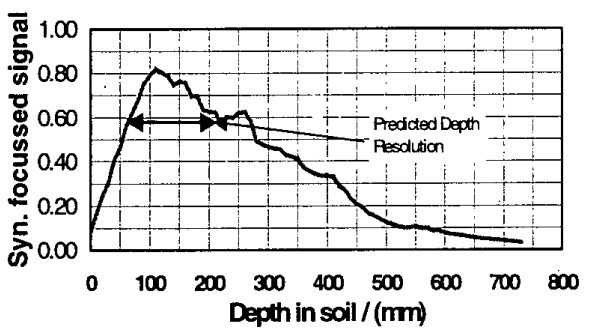

Fig 8: Vertical focussing with target at $100 \mathrm{~mm}$

These results, clearly shows the target at its actual buried location. The lateral and vertical resolution agree well with the predicted resolutions of $\lambda / \theta(\theta$ : convergence angle) and $2 \lambda$ [1] respectively for the transmit pulse used. A somewhat degraded vertical resolution is observed due to reverberations with antenna reflector (ground plane).

The performance of a PRSFSPR system in a clutter environment was investigated using a FDTD model with small randomly modelled metal blocks. The method described above was followed to obtain the reflected signals from soil with all possible transmitting elements used in separate computer runs (this was to obtain higher processing gain). Fig 9 shows the laterally focussed results with 7 paths and 25 paths with a target at $400 \mathrm{~mm}$.

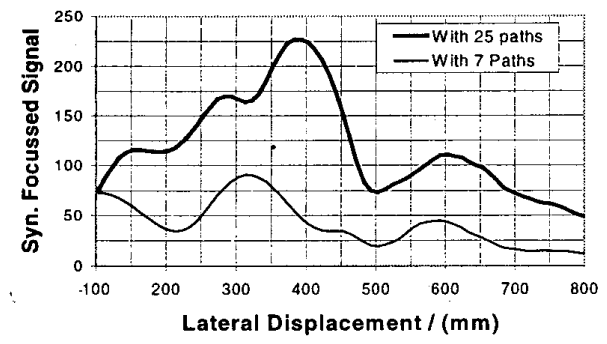

Fig 9: Lateral focussing with 7 and 25 paths

These figures demonstrate the ability of the PRSFSPR technique to detect this target in this high clutter environment.

\section{CONCLUSIONS}

A three dimensional finite-difference time-domain analysis of a surface penetrating radar which makes use of the post-reception synthetic-focusing techniques has been presented. The PRSFSPR FDTD model consists of an antenna array, feed lines, soil, the buried object and the actual antenna soil-air interface spacing. The concept of post reception synthetic focusing and its properties have been demonstrated with the FDTD results and are in good agreement with the predicted performance.

Currently the FDTD model is being further used to investigate the nature of clutter returns and various defocusing mechanisms associated with the PRSFSPR system and hence aid the development of the practical system.

\section{Acknowledgements}

The authors would like to thank the mathematical modelling group at the centre for communications research, University of Bristol for the FDTD code and their useful suggestions

\section{REFERENCES}

1. Benjamin R., 1996, Synthetic Post Reception Focusing in Near Field Radar, EUREL/IEE Conference on the detection of abandoned landmines, Edinburgh, UK.

2. Benjamin R., Post-Reception Focusing, European Patent GB 9611800.5, 6/6/1996

3. Benjamin R, Hilton G, Nilavalan R, Litobarski S, McCucheon E, 1998, Synthetically-Focused SurfacePenetrating Radar for Operation from a Moving Vehicle, EUREL/IEE Conference on the detection of abandoned landmines, Edinburgh, UK

4. Mur G, 1981, Absorbing Boundary Conditions for the Finite Difference Approximation of the Time Domain Electromagnetic Field Equations, IEEE Trans on EMC, EMC-23, No 4, 377-382.

5. Shen et al, 1990, Application of the three dimensional finite difference time domain method to the analysis of planar microstrip circuits, IEEE Trans on MTT, MTT-38, No 7, pp 849-857.

6. Hilton G S, 1993, An Investigation of Microstrip Patch Antennas and their analysis by Finite Difference Time Domain Techniques, PhD thesis, University of Bristol, UK

7. Taflove A and Umashankar 'K, 1989, Review of FDTD numerical modelling of Electro magnétic wave scattering and Radar 'Cross Section, Proc. IEEE, 77, 682-699. 